\title{
Structural and Optical Characterization of GaN/AlGaN Single Quantum Disk Nanorods
}

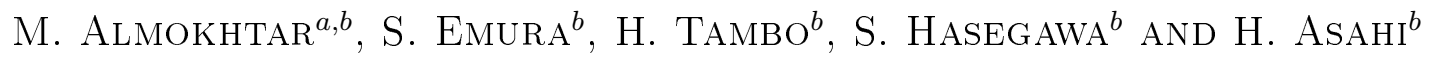 \\ ${ }^{a}$ Physics Department, Assiut University, Assiut 71516, Egypt \\ ${ }^{b}$ The Institute of Scientific and Industrial Research, Osaka University, 8-1Mihoga-oka, Ibaraki \\ Osaka 567-0047, Japan
}

\begin{abstract}
$\mathrm{GaN} / \mathrm{AlGaN}$ single quantum disks on $\mathrm{GaN}$ nanorods were grown on $\mathrm{Si}(001)$ substrate with native $\mathrm{SiO}_{2}$ layer by a plasma-assisted molecular-beam epitaxy under nitrogen-rich conditions. The transmission electron microscopy observations show single GaN nanorods images with an average thickness of $4 \mathrm{~nm}$ for the GaN single quantum disk and nanorod diameter of $15 \mathrm{~nm}$. The observed photoluminescence spectra at $8 \mathrm{~K}$ show a peak at $3.475 \mathrm{eV}$, attributed to an exciton recombination in GaN. A strong peak was observed at $3.542 \mathrm{eV}$. This peak is attributed to the quantum confinement of excitons in the GaN quantum disks.
\end{abstract}

DOI: 10.12693 /APhysPolA.123.473

PACS: 61.46.Km, 78.67.De, 81.07.Vb

\section{Introduction}

One-dimensional structures (nanowires or nanorods) of gallium nitride are known to have great prospects in novel technological applications. Because of the large band gap and structural confinement effects in such nanostructures, the fabrication of visible and UV optoelectronic devices with relatively low power consumption is potentially feasible [1-6]. It has been reported that dislocation- and strain-free GaN materials can be obtained by the forming nanoscale structures, such as columnar structures [7] and pyramidal hillocks [8]. The optical properties of GaN nanorods are mainly determined by the nanorods' dimensions, which are strongly affected by the growth parameters. However, the size distribution of the nanorods makes it difficult to analyze accurately their optical properties. Nano columnar heterostructures including quantum disks (QDisks) bring the new effects ensuing from quantum confinement effects in the columnar geometry $[9,10]$. The typical diameter of III-N nanowires grown by the plasma-assisted molecular beam epitaxy (PAMBE) techniques is in the $20-50 \mathrm{~nm}$ range, which is large compared to the GaN Bohr radius $3 \mathrm{~nm}$. Due to this weak lateral confinement, such heterostructures are coined as quantum wells, quantum dots, or quantum disks. Semiconducting nanowires exhibit phonon confinement due to the long length of the phonon wave vector $q$ compared to the radius of the nanowire. Consequently light scattering within a particle of size $D$ takes place from quasi-zone-center optical phonons with wavevector $q$ up to $\pi / D[11]$. In this study, we report on the optical properties of the self-assembled $\mathrm{Al}_{0.12} \mathrm{Ga}_{0.88} \mathrm{~N} / \mathrm{GaN} / \mathrm{Al}_{0.12} \mathrm{Ga}_{0.88} \mathrm{~N} /$ GaN nanocolumnar heterostructures comprising single GaN quantum disks (SQDisks), which were grown on $\mathrm{Si}(001)$ substrate with native $\mathrm{SiO}_{2}$ layer.

\section{Experimental}

GaN nanorods were grown on $\mathrm{Si}(001)$ substrates having native $\mathrm{SiO}_{2}$ layers by PAMBE technique. The thick- ness of the thin native $\mathrm{SiO}_{2}$ layers is less than $2 \mathrm{~nm}$. Elemental $\mathrm{Ga}, \mathrm{Al}$ and rf plasma-enhanced $\mathrm{N}_{2}$ were used as sources. After thermal cleaning of the Si substrates at $830^{\circ} \mathrm{C}$ for $15 \mathrm{~min}$, the low-temperature GaN buffer layers were first grown at $450^{\circ} \mathrm{C}$ for $40 \mathrm{~s}$. Then, annealing was performed for $10 \mathrm{~min}$ in the plasma-activated nitrogen atmosphere at $800^{\circ} \mathrm{C}$. On the next step, GaN nanorods were grown at $800^{\circ} \mathrm{C}$ followed by the growth of AlGaN, $\mathrm{GaN}$ and AlGaN layers to form the $\mathrm{AlGaN} / \mathrm{GaN} / \mathrm{AlGaN}$ SQDisks. The Ga flux $\left(1.0 \times 10^{-8}\right.$ Torr $)$ and the $\mathrm{N}_{2}$ flow rate $(1.0 \mathrm{sccm})$ with the rf plasma power $(300 \mathrm{~W})$ were fixed. The growth rate is dependent on the III $/ \mathrm{V}$ ratio, and the maximum growth rate was fixed at $1.5 \mu \mathrm{m} / \mathrm{h}$.

The photoluminescence (PL) spectra were observed using a continuous wave of a $\mathrm{He}-\mathrm{Cd}$ laser $(325 \mathrm{~nm}$ $(3.82 \mathrm{eV}))$ in the fixed power of $18 \mathrm{~mW}$ as an excitation light source. The laser-beam size is about $1 \mathrm{~mm}$. The PL light was dispersed in a $1 \mathrm{~m}$ grating monochromator (JASCO CT-100, blazed at $300 \mathrm{~nm}$ ). Both the entrance and exit slits of the monochromator are set to $1 \mathrm{~mm}$. The records of the PL intensity were carried out through a lock-in technique. An ACTI Cryogenics closed-cycle helium cryostat was used to cool the samples from room temperature (RT) down to $8 \mathrm{~K}$. A low energy pass filter was inserted in the optical line of the PL measurement to cut off the direct laser light scattered by the sample.

\section{Results and discussion}

Reflection high-energy electron diffraction (RHEED) patterns were recorded during growth for in situ monitoring of the growing surfaces. Broken-ring RHEED patterns were observed indicating that the hexagonal-GaN layers were grown with their $c$-axes perpendicular to the substrate surfaces [10]. No formation of pure metal droplets on the surfaces during growth is detected. The $\theta-2 \theta$ X-ray diffraction (XRD) rocking curves for the grown samples showed the diffraction peaks from GaN (0002) and $\mathrm{Si}(002)$. No other peaks were detected indi- 
cating that the grown nanorods have no other orientation except the $c$-axis orientation.

The formation and shape of the GaN nanorods were also confirmed using a high-resolution field emission scanning electron microscope (HR-FE-SEM). The bird's-eye view of the SQDisk nanorod structure of the GaN nanorods was shown in Fig. 1. It can be seen clearly that the vertical $c$-oriented nanorods grow perpendicular to the $\mathrm{SiO}_{2} / \mathrm{Si}$ substrate surfaces.

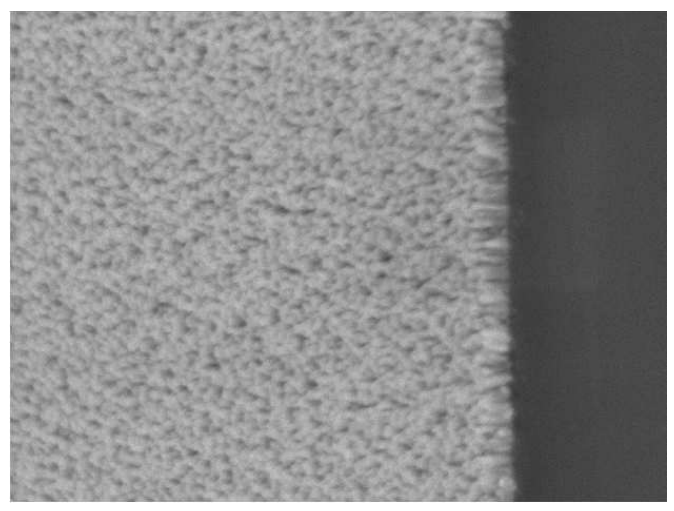

Fig. 1. Bird's-eye view by FE-SEM scans for the grown nanorods sample.

Transmission electron microscopy (TEM) observations, Fig. 2, show single GaN nanorods images with an average thickness of GaN quantum disk of $4 \mathrm{~nm}$ and nanorod diameter of $15 \mathrm{~nm}$. These structural observations using the RHEED, XRD, HR-FE-SEM and TEM show the good $c$-oriented GaN (0001) fiber texture.

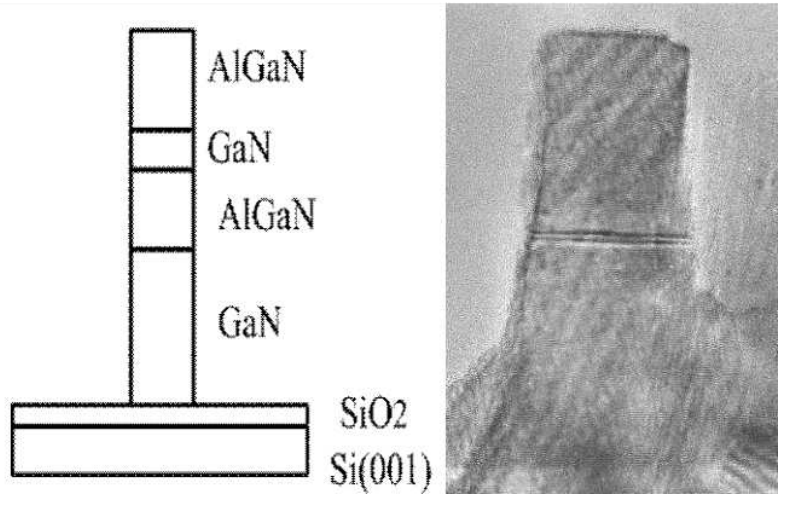

Fig. 2. The designed single quantum disk nanorod structure (a) and TEM images of AlGaN/GaN/AlGaN single quantum disk nanorods (b).

Figure 3 shows the photoluminescence (PL) spectra of the single quantum disk $\mathrm{AlGaN} / \mathrm{GaN} / \mathrm{AlGaN} / \mathrm{GaN}$ nanorods measured at $8 \mathrm{~K}$. A strong excitonic emission peak was observed at $3.475 \mathrm{eV}$. This peak can be regarded as originating from a neutral donor-bound exciton, $\mathrm{D}^{0} \mathrm{X}$ $[9,12,13]$. It was shown that this PL from the donor-bound excitons is radiated from the upper region of the
GaN nanorods without any extended structural defects and having high crystalline quality. On the higher energy side, a strong PL peak was observed at $3.542 \mathrm{eV}$, which is blue-shifted by $67 \mathrm{meV}$ from the peak energy of the standard $\mathrm{D}^{0} \mathrm{X}$ at $3.475 \mathrm{eV}$. The emission line from GaN quantum disks of $3 \mathrm{~nm}$ thicknesses was reported at $3.530 \mathrm{eV}$ at $4.2 \mathrm{~K}[14,15]$. More investigations are conducted to confirm this suggestion and will be published elsewhere. However, the peak position for AlGaN for the designed alloy with $\mathrm{Al} / \mathrm{Ga}$ ratio of $12 \%$ is expected at $3.812 \mathrm{eV}$.

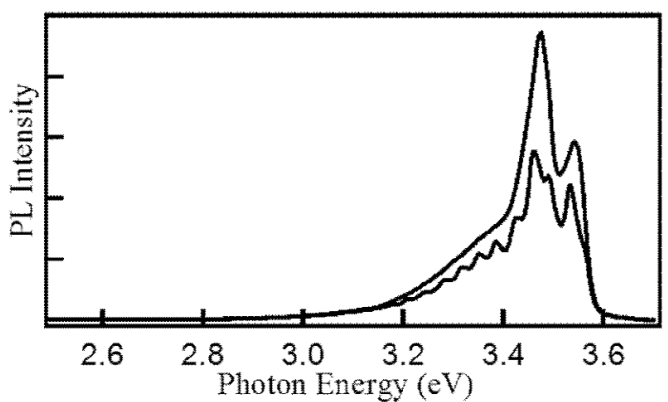

Fig. 3. PL spectrum of $\mathrm{Al}_{0.12} \mathrm{Ga}_{0.88} \mathrm{~N} / \mathrm{GaN} /$ $\mathrm{Al}_{0.12} \mathrm{Ga}_{0.88} \mathrm{~N} / \mathrm{GaN} / \mathrm{SiO}_{2} / \mathrm{Si}(001)$ measured at $8 \mathrm{~K}$, higher, and $20 \mathrm{~K}$, lower.

A broad PL peak centered around $3.35 \mathrm{eV}$, was observed in the low energy side of the donor bound energy. This observed peak energy can be associated with defect-related emissions from different regions in the nanorods and is consistent with previously reported peak values [16-22]. Experimentally, the GaN nanorods sampled by the laser spot have a range of diameters and distributed quality being excited and hence a range of defect-related bound-exciton emissions can be detected. Although strain-accommodating properties of the nanowires usually prevent the formation of dislocations, the strain induced by the lattice mismatch between $\mathrm{AlGaN}$ and $\mathrm{GaN}$ can result in the creation of point defects and line defects. Indeed, different origins for structural defects were reported in previous ensemble measurements of vertically aligned GaN nanorod arrays. Densely merged GaN nanorods on the surface may result in the formation of the localized defects. Structural defects at the $\mathrm{GaN} / \mathrm{Si}$ interface, GaN nanorods mis-oriented during growth and coalesced into GaN bundles, and stacking faults and dislocations located at the base of the nanorod in the interface region are expected. PL peaks associated with defect-related bound-exciton emissions are more dominant at low temperature. PL peak near $3.43 \mathrm{eV}$ was reported and attributed to structural defects at the nanorod/substrate interface. A broad emission (full width at half maximum around $30 \mathrm{meV}$ ) at $3.417 \mathrm{eV}$ was attributed to the recombination of excitons bound to extended structural defects located at the bottom of the nanorods produced during the initial phase of the growth. PL peak around $3.41 \mathrm{eV}$ was correlated to 
nanowire bundles presenting some degree of coalescence. $\mathrm{PL}$ emission at around $3.36 \mathrm{eV}$ was attributed to excitons bound to the structural defects at the surface.

Satellite peaks were observed in the PL spectra at $20 \mathrm{~K}$, as shown in Fig. 3. The energy separation between adjacent peaks is around $35 \mathrm{meV}$ which is smaller than the phonon replicas of longitudinal optical (LO) phonon energy in GaN (92 meV). These observed satellite peaks could be attributed to optical interference from the nanorods sample, the origin of these satellite peaks is under further investigations.

\section{Conclusions}

The GaN single quantum disk embedded in AlGaN/ GaN nanorods were grown by PAMBE techniques. The HR-FE-SEM and TEM analysis of these samples confirmed the columnar growth of the designed nanorods with the single quantum disks. The TEM images of the GaN single QDisk show an average thickness of $3 \mathrm{~nm}$ and a nanorod diameter of $15 \mathrm{~nm}$. The PL spectra observed at $8 \mathrm{~K}$ show an excitonic peak at $3.475 \mathrm{eV}$ which was attributed to exciton recombination in GaN. A strong peak was observed at $3.542 \mathrm{eV}$ and is attributed to the quantum confinement of excitons in the GaN quantum disks.

\section{Acknowledgments}

This work was supported in part by the Grant-in-Aid for Scientific Research, the Grant-in-Aid for Creative Scientific Research (contract number: 19GS1209), and the Special Education and Research Expenses from the Ministry of Education, Culture, Sports, Science and Technology (MEXT) of Japan.

\section{References}

[1] J. Renard, R. Songmuang, C. Bougerol, B. Daudin, B. Gayral, Nano Lett. 8, 2092 (2008)

[2] A. Tribu, G. Sallen, T. Aichele, R. André, J.-P. Poizat, C. Bougerol, S. Tatarenko, K. Kheng, Nano Lett. 8, 4326 (2008)

[3] F.A. Ponce, D.P. Bour, Nature 386, 351 (1997)

[4] H. Morkoc, S.N. Mohammand, Science 267, 51 (1995)

[5] C.M. Lieber, Solid State Commun. 107, 607 (1998).

[6] S. Nakamura, T. Mukai, M. Senoh, Appl. Phys. Lett. 64, 1687 (1994)
[7] I.M. Tiginyanu, V.V. Ursaki, V.V. Zalamai, S. Langa, S. Hubbard, D. Pavlidis, H. Foll, Appl. Phys. Lett. 83, 1551 (2003).

[8] L.T. Romano, T.H. Myers, Appl. Phys. Lett. 71, 3486 (1997)

[9] Y.S. Park, C.M. Park, D.J. Fu, T.W. Kang, J.E. Oh, Appl. Phys. Lett. 85, 5718 (2005).

[10] H. Tambo, S. Hasegawa, M. Uenaka, Y.K. Zhou, S. Emura, H. Asahi, Phys. Status Solidi A 208, 1576 (2011).

[11] K.W. Adu, M.D. Williams, Reber, R. Jayasingha, H.R. Gutierrez, G.U. Sumanasekera, J. Nanotechnol. 2012, 264198 (2012)

[12] E. Calleja, M.A. Sanchez-Garcia, F.J. Sanchez, F. Calle, F.B. Naranjo, E. Munoz, U. Jahn, K. Ploog, Phys. Rev. B 62, 16826 (2000)

[13] Z. Yu, S.L. Buczkowski, N.C. Giles, T.H. Myers, M.R. Richards-Babb, Appl. Phys. Lett. 69, 2731 (1996).

[14] K.H. Lee, J.H. Na, R.A. Taylor, S.N. Yi, S. Birner, Y.S. Park, C.M. Park, T.W. Kang, Appl. Phys. Lett. 89, 023103 (2006)

[15] L. Rigutti, 1, M. Tchernycheva, A. De Luna Bugallo, G. Jacopin, F.H. Julien, F. Furtmayr, M. Stutzmann, M. Eickhoff, R. Songmuang, F. Fortuna, Phys. Rev. B 81, $045411(2010)$

[16] L. Rigutti, F. Fortuna, M. Tchernycheva, A. De Luna Bugallo, G. Jacopin, F.H. Julien, S.T. Chou, Y.T. Lin, L.W. Tu, J.-C. Harmand, Phys. Status Solidi C 7, 2233 (2010)

[17] S.N. Yi, H.J. Na, K.H. Lee, A.F. Jarjour, R.A. Taylor, Y.S. Park, T.W. Kang S. Kim, D.H. Ha, G. Andrew, D. Briggs, Appl. Phys. Lett. 90, 101901 (2007)

[18] Y.S. Park, I. Hyunsik, I.T. Yoon, S.-K. Lee, Y.-H. Cho, R.A. Taylor, J. Korean Phys. Soc. 57, $756(2010)$

[19] N.A. Sanford, L.H. Robins, M.H. Gray, Y.-S. Kang, J.E. Van Nostrand, C. Stutz, R. Cortez, A.V. Davydov, A. Shapiro, I. Levin, A. Roshko, Phys. Status Solidi C 2, 2357 (2005)

[20] L.H. Robins, K.A. Bertness, J.M. Barker, N.A. Sanford, J.B. Schlager, J. Appl. Phys. 101, 113506 (2007).

[21] F. Furtmayr, M. Vielemeyer, M. Stutzmann, A. Laufer, B.K. Meyer, M. Eickhoff, J. Appl. Phys. 104, 074309 (2008)

[22] M.A. Reshchikov, H. Morkoç, J. Appl. Phys. 97, 061301 (2005) 